\title{
CHARACTERIZATIONS OF REAL HYPERSURFACES OF TYPE $A$ IN A NONFLAT COMPLEX SPACE FORM WHOSE STRUCTURE JACOBI OPERATOR IS $\xi$-PARALLEL
}

\author{
NAM-GIL KIM
}

\begin{abstract}
Let $M$ be a real hypersurface with almost contact metric structure $(\phi, \xi, \eta, g)$ of a nonflat complex space form whose structure Jacobi operator $R_{\xi}=R(\cdot, \xi) \xi$ is $\xi$-parallel. In this paper, we prove that the condition $\nabla_{\xi} R_{\xi}=0$ characterize the homogeneous real hypersurfaces of type $A$ in a complex projective space $P_{n} \mathbb{C}$ or a complex hyperbolic space $H_{n} \mathbb{C}$ when $g\left(\nabla_{\xi} \xi, \nabla_{\xi} \xi\right)$ is constant.
\end{abstract}

\section{Introduction}

A complex $n$-dimensional Kähler manifold of constant holomorphic sectional curvature $c$ is called a complex space form, which is denoted by $M_{n}(c)$. As is well-known, a complete and simply connected complex space form is complex analytically isometric to a complex projective space $P_{n} \mathbb{C}$, a complex Euclidean space $\mathbb{C}_{n}$ or a complex hyperbolic space $H_{n} \mathbb{C}$ according as $c>0, c=0$ and $c<0$.

In this paper we consider a real hypersurface $M$ in a complex space form $M_{n}(c), c \neq 0$. Then $M$ has an almost contact metric structure $(\phi, \xi, \eta, g)$ induced from the complex structure $J$ and the Kähler metric of $M_{n}(c)$. A real hypersurface is said to be a Hopf hypersurface if the structure vector field $\xi$ of $M$ is principal.

In the study of real hypersurfaces in $P_{n} \mathbb{C}$, Takagi [15] classified all homogeneous real hypersurfaces and Cecil and Ryan [3] showed that they can be regarded as the tubes of constant radius over Kähler submanifolds when the structure vector field $\xi$ is principal. Such tubes can be divided into six kinds of type $A_{1}, A_{2}, B, C, D$ and $E$.

Received May 20, 2009. Accepted June 2, 2009.

2000 Mathematics Subject Classification. Primary 53C40; Secondary 53C15.

Key words and phrases: real hypersurface, structure Jacobi operator, Ricci tensor, Hopf hypersurface.

This study was supported by the research funds from Chosun University, 2008. 
In the case of real hypersurfaces in $H_{n} \mathbb{C}$, the classification of homogeneous real hypersurfaces in $H_{n} \mathbb{C}$ is obtained by Berndt [1], Berndt and Tamaru [2]. Also such kinds of tubes are said to be real hypersurfaces of type $A_{0}, A_{1}, A_{2}$ or $B$. Among the several types of real hypersurfaces appeared in Takagi's list or Berndt's one, a particular type of tubes over totally geodesic $P_{k} \mathbb{C}$ or $H_{k} \mathbb{C}(0 \leq k \leq n-1)$ adding a horosphere in $H_{n} \mathbb{C}$, which is called type $A$, has a lot of nice geometric properties. For example, Okumura [12](resp. Montiel and Romero [11]) shows that a real hypersurface of type $\mathrm{A}$ if and only if the structure operator $\phi$ commutes with the shape operator $A \phi=\phi A$.

On the other hand, the Jacobi operator field with respect to a tangent vector field $X$ in a Riemannian manifold $M$ is defined by $R_{X}=$ $R(\cdot, X) X$, where $R$ denotes the Riemannian curvature tensor of $M$.

When we study a real hypersurface $M$ in $M_{n}(c)$, we will call the Jacobi operator on $M$ with respect to the structure vector field $\xi$ the structure Jacobi operator on $M$ and will denote it by $R_{\xi}$. But, recently it is known that there are no real hypersurfaces in $M_{n}(c)$ with parallel structure Jacobi operator $R_{\xi}$, that is $\nabla_{X} R_{\xi}=0$ for any tangent vector field $X$ on $M$ in $M_{n}(c)$ [13],[14].

Motivated by results mentioned above we consider the parallelism of the structure Jacobi operator $R_{\xi}$ in the direction of the structure vector field $\xi$, that is, $\nabla_{\xi} R_{\xi}=0$. In the previous paper [10] we prove the following:

Theorem K. Let $M$ be a real hypersurface in a nonflat complex space form $M_{n}(c), c \neq 0$ which satisfies $\mu^{2}=g\left(\nabla_{\xi} \xi, \nabla_{\xi} \xi\right)$ is constant and $\mu^{2}+c / 24 \neq 0$. Then $M$ holds $\nabla_{\xi} R_{\xi}=0$ if and only if $M$ is locally congruent to one of the following:

(1) In case that $M_{n}(c)=P_{n} \mathbb{C}$ with $\eta(A \xi) \neq 0$,

$\left(A_{1}\right)$ a geodesic hypersphere of radius $r$, where $0<r<\pi / 2$ and $r \neq \pi / 4$

$\left(A_{2}\right)$ a tube of radius $r$ over a totally geodesic $P_{k} \mathbb{C}(1 \leq k \leq n-2)$, where $0<r<\pi / 2$ and $r \neq \pi / 4$;

(2) In case that $M_{n}(c)=H_{n} \mathbb{C}$,

$\left(A_{0}\right)$ a horosphere,

$\left(A_{1}\right)$ a geodesic hypersphere or a tube over a complex hyperbolic hyperplane $H_{n-1} \mathbb{C}$,

$\left(A_{2}\right)$ a tube over a totally geodesic $H_{k} \mathbb{C}(1 \leq k \leq n-2)$. 
The main purpose of the present paper is to improve Theorem $\mathrm{K}$ without the condition $\mu^{2}+c / 24 \neq 0$. As an application of this result, we give another simple proof of the following theorem:

Theorem $\mathbf{L}([\mathbf{8}])$. Let $M$ be a real hypersurface in a complex space form $M_{n}(c), c \neq 0$ satisfying $\nabla_{\xi} S=0$ and $\nabla_{\xi} R_{\xi}=0$. Then $M$ is a Hopf hypersurface in $M_{n}(c)$, where $S$ denotes the Ricci tensor of $M$.

\section{Preliminaries}

Let $M$ be a real hypersurface immersed in a complex space form $\left(M_{n}(c), G\right)$ with almost complex structure $J$, and the Kähler metric $G$ of constant holomorphic sectional curvature $c$, and let $N$ be a unit normal vector field on $M$. The Riemannian connection $\tilde{\nabla}$ in $M_{n}(c)$ and that $\nabla$ in $M$ are related by the following formulas for any vector fields $X$ and $Y$ on $M$ :

$$
\tilde{\nabla}_{Y} X=\nabla_{Y} X+g(A Y, X) N, \quad \tilde{\nabla}_{X} N=-A X,
$$

where $g$ denotes the Riemannian metric on $M$ induced from that $G$ of $M_{n}(c)$ and $A$ is the shape operator in the direction of $N$ in $M_{n}(c)$. For any vector field $X$ tangent to $M$, we put

$$
J X=\phi X+\eta(X) N, \quad J N=-\xi .
$$

Then we may see that the structure $(\phi, \xi, \eta, g)$ is an almost contact metric structure on $M$, namely, we have

$$
\begin{aligned}
& \phi^{2} X=-X+\eta(X) \xi, \quad g(\phi X, \phi Y)=g(X, Y)-\eta(X) \eta(Y), \\
& \eta(\xi)=1, \quad \phi \xi=0, \quad \eta(X)=g(X, \xi)
\end{aligned}
$$

for any vector fields $X$ and $Y$ on $M$.

From the fact $\tilde{\nabla} J=0$, and above equations, we verify that

$$
\left(\nabla_{X} \phi\right) Y=\eta(Y) A X-g(A X, Y) \xi, \quad \nabla_{X} \xi=\phi A X .
$$

Since we consider that the ambient manifold is of constant holomorphic sectional curvature $c$, we have the following Gauss and Codazzi equations respectively.

$$
\begin{aligned}
R(X, Y) Z= & \frac{c}{4}\{g(Y, Z) X-g(X, Z) Y+g(\phi Y, Z) \phi X \\
& -g(\phi X, Z) \phi Y+2 g(\phi X, Y) \phi Z\} \\
& +g(A Y, Z) A X-g(A X, Z) A Y
\end{aligned}
$$

$$
\left(\nabla_{X} A\right) Y-\left(\nabla_{X} A\right) X=\frac{c}{4}\{\eta(X) \phi Y-\eta(Y) \phi X-2 g(\phi X, Y) \xi\}
$$


for any tangent vector fields $X, Y$ and $Z$ on $M$, where $R$ denote the Riemann-Christoffel curvature tensor of $M$. We shall denote the Ricci tensor of type $(1,1)$ by $S$. Then it follows from $(2.2)$ that

$$
S X=\frac{c}{4}\{(2 n+1) X-3 \eta(X) \xi\}+h A X-A^{2} X,
$$

where $h=$ trace $A$. Further, using (2.1), we obtain

$$
\begin{gathered}
\left(\nabla_{X} S\right) Y=-\frac{3}{4} c\{g(\phi A X, Y) \xi+\eta(Y) \phi A X\}+(X h) A Y \\
+(h I-A)\left(\nabla_{X} A\right) Y-\left(\nabla_{X} A\right) A Y,
\end{gathered}
$$

where $I$ is the identity map.

In what follows, to write our formulas in convention forms, we denote $\alpha=\eta(A \xi), \beta=\eta\left(A^{2} \xi\right)$, and for a function $f$ we denote by $\nabla f$ the gradient vector field of $f$.

We put $U=\nabla_{\xi} \xi$, then $U$ is orthogonal to the structure vector field $\xi$. Thus, it is, using (2.1), seen that

$$
\phi U=-A \xi+\alpha \xi,
$$

which enables us to obtain $g(U, U)=\beta-\alpha^{2}$. Thus we easily see that $\xi$ is a principal curvature vector, that is $A \xi=\alpha \xi$ if and only $\beta-\alpha^{2}=0$.

Now, differentiating (2.6) covariantly along $M$ and using (2.1), we find

$$
\begin{gathered}
\eta(X) g(A U+\nabla \alpha, Y)+g\left(\phi X, \nabla_{Y} U\right) \\
=g\left(\left(\nabla_{Y} A\right) X, \xi\right)-g(A \phi A X, Y)+\alpha g(A \phi X, Y),
\end{gathered}
$$

which shows that

$$
\left(\nabla_{\xi} A\right) \xi=2 A U+\nabla \alpha
$$

because of (2.3). From (2.7) we also have

$$
\nabla_{\xi} U=3 \phi A U+\alpha A \xi-\beta \xi+\phi \nabla \alpha,
$$

where we have used (2.1).

If $\beta-\alpha^{2} \neq 0$, then we can put

$$
A \xi=\alpha \xi+\mu W,
$$

where $W$ is a unit vector field orthogonal to $\xi$. Then by (2.1) we see that $U=\mu \phi W$ and hence $g(U, U)=\mu^{2}$, and $W$ is also orthogonal to $U$. Thus, we verify, making use of (2.6) and (2.10), that

$$
\begin{aligned}
& \mu g\left(\nabla_{X} W, \xi\right)=g(A U, X), \\
& g\left(\nabla_{X} \xi, U\right)=\mu g(A W, X) .
\end{aligned}
$$


The Gauss equation (2.2) gives the structure Jacobi operator $R_{\xi}$ :

$$
R_{\xi} X=R(X, \xi) \xi=\frac{c}{4}\{X-\eta(X) \xi\}+\alpha A X-\eta(A X) A \xi
$$

for any vector field $X$ on $M$.

\section{Real hypersurfaces satisfying $\nabla_{\xi} R_{\xi}=0$}

In this section the elemental factors of real hypersurfaces satisfying $\nabla_{\xi} R_{\xi}=0$ in $M_{n}(c)$ are recalled ([10]). We set $\Omega=\{p \in M \mid \mu(p) \neq 0\}$ and suppose that $\Omega \neq \varnothing$, that is $\xi$ is not principal curvature vector on $M$. Hereafter, unless otherwise stated, we discuss our discussions on the open set $\Omega$ of $M$.

Differentiating (2.13) covariantly, and using (2.8), we find

$$
\begin{aligned}
g\left(\left(\nabla_{\xi} R_{\xi}\right) Y, Z\right)= & -\frac{c}{4}\{u(Y) \eta(Z)+u(Z) \eta(Y)\}+(\xi \alpha) g(A Y, Z) \\
& +\alpha g\left(\left(\nabla_{\xi} A\right) Y, Z\right)-\eta(A Z)\{3 g(A U, Y)+Y \alpha\} \\
& -\eta(A Y)\{3 g(A U, Z)+Z \alpha\},
\end{aligned}
$$

where $u$ is a 1 -form dual to $U$ with respect to $g$, that is $u(X)=g(U, X)$.

We assume that $\nabla_{\xi} R_{\xi}=0$. Then from (3.1) we have

$$
\begin{gathered}
\alpha\left(\nabla_{\xi} A\right) X+(\xi \alpha) A X=\frac{c}{4}(u(X) \xi+\eta(X) U)+\eta(A X)(3 A U+\nabla \alpha) \\
+\{3 g(A U, X)+X \alpha\} A \xi .
\end{gathered}
$$

Putting $X=\xi$ in this and using (2.8), we find

$$
\alpha A U+\frac{c}{4} U=0
$$

which shows that $\alpha \neq 0$ on $\Omega$.

Differentiating (3.3) covariantly and using itself, we obtain

$$
-\frac{c}{4}(X \alpha) U+\alpha^{2}\left(\nabla_{X} A\right) U+\alpha^{2} A \nabla_{X} U+\frac{c}{4} \alpha \nabla_{X} U=0,
$$

or, using (2.3) and (2.6)

$$
\begin{gathered}
\frac{c}{4}\{(Y \alpha) u(X)-(X \alpha) u(Y)\}+\frac{c}{4} \alpha^{2} \mu\{\eta(X) w(Y)-\eta(Y) w(X)\} \\
+\alpha^{2}\left\{g\left(A \nabla_{X} U, Y\right)-g\left(A \nabla_{Y} U, X\right)\right\}+\frac{c}{4} \alpha d u(X, Y)=0,
\end{gathered}
$$

where $w$ is a dual 1 -form of $W$ with respect to $g$, that is $w(X)=$ $g(W, X)$. Here, $d u$ is the exterior derivative of a 1 -form $u$ given by

$$
d u(X, Y)=X u(Y)-Y u(X)-u([X, Y]) .
$$

If we replace $X$ by $U$ in (3.5), then we have

$$
\frac{c}{4}\left\{\mu^{2} \nabla \alpha-(U \alpha) U\right\}+\alpha^{2} A \nabla_{U} U+\frac{c}{4} \alpha \nabla_{U} U=0,
$$


because $U$ and $W$ are mutually orthogonal.

Combining (2.7) to (3.2) and using (2.3), we obtain

$$
\begin{aligned}
\alpha^{2} \phi \nabla_{X} U= & \alpha^{2}(X \alpha) \xi-\frac{c}{4} \alpha u(X) \xi+\alpha(\xi \alpha) A X+\frac{c}{4} \alpha^{2} \phi X \\
& -\eta(A X)\left(\alpha \nabla \alpha-\frac{3}{4} c U\right)-\left\{\alpha(X \alpha)-\frac{3}{4} c u(X)\right\} A \xi \\
& -\frac{c}{4} \alpha\{u(X) \xi+\eta(X) U\}-\alpha^{2} A \phi A X+\alpha^{3} \phi A X .
\end{aligned}
$$

Applying $\phi$ to this and using (2.12), we have

$$
\begin{gathered}
\alpha^{2} \nabla_{X} U+\alpha^{2} \mu g(A W, X) \xi-\alpha \eta(A X) \phi \nabla \alpha \\
=-\alpha(\xi \alpha) \phi A X+\frac{c}{4} \alpha^{2}\{X-\eta(X) \xi\}+\frac{3}{4} c \mu \eta(A X) W+\alpha(X \alpha) U \\
-\frac{3}{4} c u(X) U+\alpha^{3} A X-\frac{c}{4} \alpha \mu \eta(X) W-\alpha^{3} \eta(A X) \xi+\alpha^{2} \phi A \phi A X .
\end{gathered}
$$

On the other hand, differentiating (2.10) covariantly and using (2.1), we find

$$
\left(\nabla_{X} A\right) \xi+A \phi A X=(X \alpha) \xi+\alpha \phi A X+(X \mu) W+\mu \nabla_{X} W,
$$

or, using (2.3) and (3.2),

$$
\begin{aligned}
\alpha \mu \nabla_{X} W= & \frac{c}{4}(u(X) \xi+\eta(X) U)+\eta(A X)(3 A U+\nabla \alpha) \\
& +3 g(A U, X) A \xi+\mu(X \alpha) W-\frac{c}{4} \alpha \phi X \\
& +\alpha A \phi A X-\alpha^{2} \phi A X-(\xi \alpha) A X-\alpha(X \mu) W .
\end{aligned}
$$

Putting $X=\alpha U$ in (3.2) and taking account of (3.3), we find

$$
\alpha^{2}\left(\nabla_{\xi} A\right) U-\frac{c}{4}(\xi \alpha) U=\frac{c}{4} \alpha \mu^{2} \xi+\left\{\alpha(U \alpha)-\frac{3}{4} c \mu^{2}\right\} A \xi .
$$

Because of (3.3), the equation (2.9) turns out to be

$$
\alpha \nabla_{\xi} U=\frac{3}{4} c \mu W+\alpha^{2} A \xi-\alpha \beta \xi+\alpha \phi \nabla \alpha .
$$

If we replace $X=\alpha \xi$ in (3.4) and make use of (3.3) and the last two equations, we obtain

$$
\begin{gathered}
\alpha A \phi \nabla \alpha+\frac{c}{4} \phi \nabla \alpha+(U \alpha) A \xi \\
+\mu\left(\alpha^{2}+\frac{3}{4} c\right)\left\{A W-\mu \xi-\frac{1}{\alpha}\left(\mu^{2}-\frac{c}{4}\right) W\right\}=0 .
\end{gathered}
$$

4. Real hypersurfaces satisfying $g\left(\nabla_{\xi} \xi, \nabla_{\xi} \xi\right)$ is constant

In this section we assume that $\nabla_{\xi} R_{\xi}=0$ and at the same time $g\left(\nabla_{\xi} \xi, \nabla_{\xi} \xi\right)$ is constant, that is, $g(U, U)=\mu^{2}$ is constant. Then we obtain $\nabla \mu=0$ on $\Omega$, which implies that $g\left(\nabla_{X} U, U\right)=0$. Thus, taking an inner product $U$ to $(3.8)$, we get

$$
(W \alpha) A \xi=(\xi \alpha) A W+\frac{3}{4 \alpha} c \mu U-\mu \nabla \alpha+\alpha A \phi A W,
$$


which shows that $W \alpha=0$ and $(\xi \alpha) g(A W, W)=0$ because of (3.3). Thus, it follows that

$$
\mu\left(\nabla \alpha-\frac{3}{4 \alpha} c U\right)=(\xi \alpha) A W+\alpha A \phi A W,
$$

which implies that

$$
\alpha(U \alpha)=\frac{c}{4}\left(3 \mu^{2}-\alpha \lambda\right),
$$

where we have put $\lambda=g(A W, W)$.

Putting $X=U$ in (3.8) and using (3.3) and (4.2), we find

$$
\alpha \nabla_{U} U=-\frac{c}{4 \alpha} \mu(\xi \alpha) W-\frac{c}{4} \lambda U+\frac{c}{4} \mu \phi A W .
$$

If we take account of (3.3) and this, then (3.6) turns out to be

$$
\mu^{2} \nabla \alpha-(U \alpha) U=\frac{\mu}{\alpha}(\xi \alpha)\left(\alpha A W+\frac{c}{4} W\right)-\mu\left(\alpha A \phi A W+\frac{c}{4} \phi A W\right),
$$

which together with $\lambda(\xi \alpha)=0$ gives $\xi \alpha=0$. So we see, using (4.2), that

$$
\mu \nabla \alpha=\left(\frac{3}{4 \alpha} c \mu-\frac{c \lambda}{4 \mu}\right) U-\alpha A \phi A W-\frac{c}{4} \phi A W .
$$

Combining this to (4.1), we obtain

$$
\alpha A \phi A W=-\frac{c}{8}\left(\frac{\lambda}{\mu} U+\phi A W\right)
$$

because of $\xi \alpha=0$. Therefore, (4.3) is reduced to

$$
\mu\left(\alpha \nabla \alpha-\frac{3}{4} c U\right)=-\frac{c}{8}\left(\alpha \phi A W+\frac{\alpha \lambda}{\mu} U\right) .
$$

From this, we can verify that

Remark 1. $\alpha=$ constant on $\Omega$ (see, Lemma 3 of [10]).

Using $\alpha=$ constant on $\Omega$, we see from (4.2) that

$$
\alpha \lambda=3 \mu^{2} .
$$

Hence, (4.4) shows that $\lambda U=\mu \phi A W$. So we have

$$
A W=\mu \xi+\lambda W .
$$

Differentiating this covariantly, we find

$$
\left(\nabla_{X} A\right) W+A \nabla_{X} W=\mu \phi A X+\lambda \nabla_{X} W
$$


because $\lambda$ is constant by virtue of Remark 1 and (4.5). Thus we have a Codazzi-type formula for $w$ :

$$
\begin{gathered}
\lambda\left(\nabla_{X} w\right)(Y)-\left(\nabla_{Y} w\right)(X) \\
=\frac{c}{4 \mu}(u(X) \eta(Y)-u(Y) \eta(X))+g\left(A \nabla_{X} W, Y\right) \\
-g\left(A \nabla_{Y} W, X\right)-\mu g((\phi A+A \phi) X, Y),
\end{gathered}
$$

where we have used (2.3).

On the other hand, replacing $X$ by $U$ in (3.9) and using (3.3), (4.6) and Remark 1, we find

$$
\alpha \nabla_{U} W=-\frac{c}{4} \mu \xi
$$

In the same way, we also have

$$
\alpha g\left(\nabla_{X} W, U\right)=-\mu\left(\alpha^{2}+\frac{3}{4} c\right) \eta(X)-\left(\frac{c}{4} \alpha+\frac{c}{2} \lambda+\alpha^{2} \lambda\right) w(X) .
$$

If we replace $X$ by $U$ in (4.7) and make use of (3.3) and (4.8), then we obtain

$$
\left(\lambda+\frac{c}{4 \alpha}\right) g\left(\nabla_{X} W, U\right)=-\mu^{2} g(A W, X)+\frac{c}{2 \alpha} \mu^{2} w(X)-\frac{c}{4 \alpha} \lambda \mu \eta(X),
$$

which together with (4.9) yields

$$
\begin{aligned}
& \left(\lambda+\frac{c}{4 \alpha}\right)\left\{\mu\left(\alpha^{2}+\frac{3}{4} c\right) \xi+\left(\frac{c}{2} \alpha+\frac{c}{2} \lambda+\alpha^{2} \lambda\right) W\right\} \\
= & \mu^{2} \alpha A W-\frac{c}{2} \mu^{2} W+\frac{c}{4} \lambda \mu \xi .
\end{aligned}
$$

Using (4.6), we see from this

$$
\left(\lambda+\frac{c}{4 \alpha}\right)\left(\alpha^{2}+\frac{3}{4} c\right)-\alpha \mu^{2}-\frac{c}{4} \lambda=0
$$

and

$$
\left(\lambda+\frac{c}{4 \alpha}\right)\left(\frac{c}{4} \alpha+\frac{c}{2} \lambda+\alpha^{2} \lambda\right)+\mu^{2}\left(\frac{c}{2}-\alpha \lambda\right)=0 .
$$

Combining (4.10) to (4.5), we verify that $\left(\alpha^{2}+\frac{3}{4} c\right)\left(2 \mu^{2}+\frac{c}{4}\right)=0$, which together with (4.11) implies that

$$
\alpha^{2}+\frac{3}{4} c=0
$$

From this and (4.5) we have

$$
\mu^{2} \alpha=-\frac{c}{4} \lambda,
$$


which connected to (4.11) gives $6 \lambda=\alpha$. So we see, using (4.13), that (for detail, see [?])

$$
6 \mu^{2}+\frac{c}{4}=0
$$

Using (3.3) and (4.12), the equation (3.2) can be written as

$$
\left(\nabla_{\xi} A\right) X=\frac{c}{4 \alpha}\{u(X) \xi+\eta(X) U\}+\eta(A X) U+u(X) A \xi,
$$

which shows that

$$
\left(\nabla_{\xi} A\right) W=\mu U
$$

Because of (3.3) and (4.12), the equation (3.9) turns out to be

$$
\begin{aligned}
& \mu \nabla_{X} W=\mu(u(X) W+w(X) U)-\frac{c}{2 \alpha}(u(X) \xi+\eta(X) U)-\frac{c}{4} \phi X \\
& +A \phi A X-\alpha \phi A X,
\end{aligned}
$$

which implies that

$$
\begin{aligned}
\mu\left\{\left(\nabla_{X} w\right) Y-\left(\nabla_{Y} w\right) X\right\}= & -\frac{c}{2} g(\phi X, Y)+2 g(A \phi A X, Y) \\
& -\alpha g((A \phi-\phi A) X, Y) .
\end{aligned}
$$

Putting $X=U$ in this and taking account of (3.3) and (4.12), we find

$$
\left(\nabla_{U} w\right) Y-\left(\nabla_{Y} w\right) U=\frac{c}{4}\left(w(Y)-\frac{1}{\alpha} g(A W, Y)\right) .
$$

If we take account of (4.6) and (4.12), then (3.8) becomes

$$
\nabla_{X} U+\mu g(A \xi, X) \xi+g(A \xi, X) A \xi
$$

$\stackrel{(4.19)}{=} \alpha A X+\frac{c}{4}(X-\eta(X) \xi)+u(X) U-\frac{c}{4 \alpha} \mu \eta(X) W+\phi A \phi A X$.

Replacing $X$ by $U$ in this and making use of (3.3), (4.6) and (4.12), we obtain

$$
\nabla_{U} U=0
$$

Thus, (3.4) implies that

$$
\left(\nabla_{U} A\right) U=0
$$

by virtue of (4.12).

From (2.3), (3.4) and (4.12) we have $\frac{c}{4} \mu(\eta(X) w(Y)-\eta(Y) w(X))+\alpha\left\{g\left(A \nabla_{X} U, Y\right)-g\left(A \nabla_{Y} U, X\right)\right\}+\frac{c}{4} d u(X, Y)=0$, or, using (4.19)

$$
d u(X, Y)=\mu \lambda(\eta(Y) w(X)-\eta(X) w(Y)) .
$$


Using above equations, we are now going to prove that $\Omega=\varnothing$.

In the following, let $\left\{e_{1}, \ldots, e_{2 n-1}\right\}$ be an orthonormal frame field of $M$. And the coefficients of tensors are defined by $\xi^{i}=g\left(\xi, e_{i}\right), A_{i}{ }^{j}=$ $g\left(A e_{i}, e_{j}\right), \phi_{i}{ }^{j}=g\left(\phi e_{i}, e_{j}\right), \nabla_{i} U^{j}=g\left(\nabla_{e_{i}} U, e_{j}\right)$ and so on. Furthermore, we use the Einstein rule, that is, we take the summation for the same indices in the equation. So (4.17) can be written as

$$
\begin{aligned}
\mu \nabla_{j} W_{i}=\left(\mu W_{i}-\frac{c}{2 \alpha} \xi_{i}\right) U_{j} & +\left(\mu W_{j}-\frac{c}{2 \alpha} \xi_{j}\right) U_{i}+\alpha A_{j r} \phi_{i}{ }^{r}-\frac{c}{4} \phi_{j i} \\
& -A_{i}{ }^{r} A_{j s} \phi_{r}{ }^{s} .
\end{aligned}
$$

Differentiating this covariantly and using (2.1), we find

$$
\begin{aligned}
\mu \nabla_{k} \nabla_{j} W_{i}= & \left(\mu \nabla_{k} W_{i}+\frac{c}{2 \alpha} A_{k r} \phi_{i}{ }^{r}\right) U_{j}+\left(\mu W_{i}-\frac{c}{2 \alpha} \xi_{i}\right) \nabla_{k} U_{j} \\
& +\left(\mu \nabla_{k} W_{j}+\frac{c}{2 \alpha} A_{k r} \phi_{j}{ }^{r}\right) U_{i}+\left(\mu W_{j}-\frac{c}{2 \alpha} \xi_{j}\right) \nabla_{k} U_{i} \\
& +\alpha\left(\nabla_{k} A_{j r}\right) \phi_{i}{ }^{r}+\alpha \xi_{i} A_{j k}{ }^{2}-\alpha\left(A_{j r} \xi^{r}\right) A_{k i}+\frac{c}{4}\left(\xi_{i} A_{k j}-\xi_{j} A_{k i}\right) \\
& -\left(\nabla_{k} A_{i}{ }^{r}\right) A_{j s} \phi_{i}{ }^{s}-A_{i}{ }^{r}\left(\nabla_{k} A_{j s}\right) \phi_{r}{ }^{s}+\left(A_{j r} \xi^{r}\right) A_{k i}{ }^{2}-\left(A_{i r} \xi^{r}\right) A_{k j}{ }^{2},
\end{aligned}
$$

where we have used (4.2) and $\mu=$ const. If we take the symmetric part with respect to indices $k$ and $j$ in this, and make use of (2.3), (4.22) and the Ricci-identity, we obtain

$$
\begin{aligned}
-\mu R_{k j i h} W^{h}= & \left(\mu \nabla_{k} W_{i}+\frac{c}{2 \alpha} A_{k r} \phi_{i}{ }^{r}\right) U_{j}-\left(\mu \nabla_{j} W_{i}+\frac{c}{2 \alpha} A_{j r} \phi_{i}{ }^{r}\right) U_{k} \\
& +\left\{\mu\left(\nabla_{k} W_{j}-\nabla_{j} W_{k}\right)+\frac{c}{2 \alpha}\left(A_{k r} \phi_{j}{ }^{r}-A_{j r} \phi_{k}{ }^{r}\right)\right\} U_{i} \\
& +\mu \lambda\left(\mu W_{i}-\frac{c}{2 \alpha} \xi_{i}\right)\left(\xi_{k} W_{j}-\xi_{j} W_{k}\right)+\frac{c}{4} \alpha\left(\xi_{k} g_{j i}-\xi_{j} g_{k i}\right) \\
& +\left(\mu W_{i}-\frac{c}{2 \alpha} \xi_{i}\right) \nabla_{k} U_{i}-\left(\mu W_{k}-\frac{c}{2 \alpha} \xi_{k}\right) \nabla_{j} U_{i} \\
& +A_{k r} \xi^{r}\left(\alpha A_{j i}-A_{j i}{ }^{2}\right)-A_{j r} \xi^{r}\left(\alpha A_{k i}-A_{k i}{ }^{2}\right) \\
& +\left(\nabla_{j} A_{i}{ }^{r}\right) A_{k s} \phi_{r}{ }^{s}-\left(\nabla_{k} A_{i}{ }^{r}\right) A_{j s} \phi_{r}{ }^{s} .
\end{aligned}
$$

On the other hand from (2.2) we have

$$
R_{k j i h} U^{k} W^{h}=-\frac{c}{4}\left(3 W_{j} U_{i}+W_{i} U_{j}+\mu \phi_{j i}\right)+\frac{c}{4 \alpha} U_{i} A_{j r} W^{r},
$$

where we have used (2.6), (2.10) and (3.3). 
Multiplying $U^{k}$ to (4.24) and summing for $k$, and using (3.3), (4.8), (4.12), (4.18) and the last equation, we have

$$
\begin{aligned}
\frac{c}{12} \mu & \left(U_{j} W_{i}+U_{i} W_{j}\right)+\frac{c}{4} \mu^{2} \phi_{j i}+\mu^{2}\left(\mu \nabla_{j} W_{i}+\frac{c}{2 \alpha} A_{j r} \phi_{i}^{r}\right) \\
& =-\frac{c}{4 \alpha} \mu^{2} \xi_{i} U_{j}+\left(\frac{c}{2 \alpha} \mu A_{j r} W^{r}-\frac{c}{12} A_{j r} \xi^{r}\right) U_{i}-\frac{c}{4 \alpha} \mu\left(\nabla_{j} A_{i r}\right) W^{r} \\
& -\left(\nabla_{U} A_{i}{ }^{r}\right) A_{j s} \phi_{r}{ }^{s} .
\end{aligned}
$$

Since we see, using (4.23), that $\nabla_{\xi} W=0$, if we multiply $\xi^{j}$ to this and summing for $j$, we obtain $\mu^{2}+\frac{c}{12}=0$, where we have used (4.12), (4.16) and (4.21). This contradicts (4.14). Hence we verify that $\Omega=\varnothing$. Thus, we have

Lemma 1. Let $M$ be a real hypersurface satisfies $\nabla_{\xi} R_{\xi}=0$ and $g\left(\nabla_{\xi} \xi, \nabla_{\xi} \xi\right)=$ const. in $M_{n}(c), c \neq 0$. Then $M$ is a Hopf hypersurface in $M_{n}(c)$.

According to Lemma 1 , we have $A \xi=\alpha \xi$ on $M$. So we see in addition that $\alpha$ is constant on $M([9])$. Thus, we, using (3.2), verify that $\alpha \nabla_{\xi} A=$ 0 , which together with (2.1) and (2.3) implies that $\alpha(A \phi-\phi A)=0$.

Here, we note the case $\alpha=0$ corresponds to the case of tube of radius $\frac{\pi}{4}$ in $P_{n} \mathbb{C}$ (see [3]). But, in the case of $H_{n} \mathbb{C}$ it is known that $\alpha$ never vanishes for Hopf hypersurfaces (cf. [1]). Owing to Okumura [12] or Montiel and Romero [11] stated in Introduction, we have

Theorem 2. Let $M$ be a real hypersurface in a complex space form $M_{n}(c), c \neq 0$ which satisfies $g\left(\nabla_{\xi} \xi, \nabla_{\xi} \xi\right)$ is constant. Then $M$ holds $\nabla_{\xi} R_{\xi}=0$ if and only if $M$ is locally congruent to one of the following:

(I) In case that $M_{n}(c)=P_{n} \mathbb{C}$ with $\eta(A \xi) \neq 0$, $r \neq \pi / 4$,

$\left(A_{1}\right)$ a geodesic hypersphere of radius $r$, where $0<r<\pi / 2$ and

$\left(A_{2}\right)$ a tube of radius $r$ over a totally geodesic $P_{k} \mathbb{C}(1 \leq k \leq n-2)$, where $0<r<\pi / 2$ and $r \neq \pi / 4$.

(II) In case that $M_{n}(c)=H_{n} \mathbb{C}$,

$\left(A_{0}\right)$ a horosphere;

$\left(A_{1}\right)$ a geodesic hypersphere or a tube over a complex hyperbolic hyperplane $H_{n-1} \mathbb{C}$;

$\left(A_{2}\right)$ a tube over a totally geodesic $H_{k} \mathbb{C}(1 \leq k \leq n-2)$.

\section{Proof of Theorem L}


Let $M$ be a real hypersurface in $M_{n}(c), c \neq 0$ satisfying $\nabla_{\xi} R_{\xi}=0$. Then $(3.1) \sim(3.10)$ are valid on $\Omega$.

Further, we assume $\nabla_{\xi} S=0$ on $M$. Then replacing $X$ by $\xi$ in (2.5) and using the Codazzi equation (2.3), we obtain

$$
\begin{aligned}
\frac{3}{4} c\{u(X) & \eta(Y)+u(Y) \eta(X)\}+\frac{c}{4}\{g(A Y, \phi X)+g(A X, \phi Y)\} \\
& =(\xi h) g(A X, Y)+h g\left(\left(\nabla_{Y} A\right) X, \xi\right)-\frac{c}{4} h g(\phi X, Y) \\
& -g\left(A Y,\left(\nabla_{X} A\right) \xi\right)-g\left(A X,\left(\nabla_{Y} A\right) \xi\right) .
\end{aligned}
$$

Combining (5.1) to (3.2) and making use of (3.3), we find

$$
\begin{aligned}
& \frac{c}{4}\left(3 \alpha^{2}-h \alpha-\frac{c}{4}\right)(u(X) \xi+\eta(X) U)-2 \alpha(\xi \alpha) A^{2} X+\alpha\{h(\xi \alpha)-\alpha(\xi h)\} A X \\
& \quad=g(A \xi, X)\left\{h \alpha \nabla \alpha-\alpha A \nabla \alpha+\frac{3}{4} c(A U-h U)-\frac{c}{4} \alpha U\right\} \\
& \quad+\left\{h \alpha(X \alpha)-\alpha g(A \nabla \alpha, X)+\frac{3}{4} c(g(A U, X)-h u(X))-\frac{c}{4} \alpha u(X)\right\} A \xi \\
& \quad+\left\{\frac{3}{4} c u(X)-\alpha(X \alpha)\right\} A^{2} \xi+\left\{\frac{3}{4} c U-\alpha \nabla \alpha\right\} g\left(A^{2} \xi, X\right) .
\end{aligned}
$$

Since $U$ is orthogonal to $\xi$, we see, using (2.10) and (3.3), that $g\left(A^{2} \xi, U\right)=0$, by replacing $X$ by $U$ in (5.2) and taking account of (3.3), we get

$$
\begin{aligned}
& \left\{\frac{3}{4} c \mu^{2}-\alpha(U \alpha)\right\} A^{2} \xi+\left\{\left(h \alpha+\frac{c}{4}\right) U \alpha-\frac{3}{4} c\left(\frac{c}{4 \alpha}+h\right) \mu^{2}-\frac{c}{4} \alpha \mu^{2}\right\} A \xi \\
& =\frac{c}{4}\left(3 \alpha^{2}-h \alpha-\frac{c}{4}\right) \mu^{2} \xi-\frac{c}{4}\left\{\left(\frac{c}{2 \alpha}+h\right) \xi \alpha-\alpha(\xi h)\right\} U,
\end{aligned}
$$

which shows that

$$
\left(h \alpha+\frac{c}{2}\right) \xi \alpha-\alpha^{2}(\xi h)=0 .
$$

We notice here that $\alpha(U \alpha)-\frac{3}{4} c \mu^{2} \neq 0$ on $\Omega$. In fact, if not, then we have $\alpha A \xi=\left(h \alpha+\frac{c}{4}-3 \alpha^{2}\right) \xi$ because of (5.3) and (5.4). So we have $A \xi=\alpha \xi$, a contradiction. Thus, $\alpha(U \alpha)-\frac{3}{4} c \mu^{2} \neq 0$ is satisfied everywhere. Therefore, we have from (5.3) and (5.4) the following:

$$
A^{2} \xi=\rho A \xi+(\beta-\rho \alpha) \xi,
$$

where the function $\rho$ is defined by $\mu \rho=g\left(A^{2} \xi, W\right)$. 
Combining (2.10) to (5.5), we verify that

$$
A W=\mu \xi+\lambda W
$$

because of $\mu \neq 0$, where we have put $\lambda=\rho-\alpha$.

If we take an inner product (3.10) with $W$ and make use of (5.6), then we obtain

$$
\left(\beta-\rho \alpha-\frac{c}{4}\right)\left\{\alpha(U \alpha)-\mu^{2}\left(\alpha^{2}+\frac{3}{4} c\right)\right\}=0 .
$$

Remark 2. It is proved in [5] that $\Omega=\varnothing$ if a real hypersurface $M$ satisfies $\nabla_{\xi} R_{\xi}=0$ and $A R_{\xi}-R_{\xi} A=0$ in $M_{n}(c), c \neq 0$.

From (5.7) we have

$$
\alpha(U \alpha)=\mu^{2}\left(\alpha^{2}+\frac{3}{4} c\right) .
$$

Indeed, if not, we have $\beta-\rho \alpha=\frac{c}{4}$. Thus, (5.5) becomes $A^{2} \xi=\rho A \xi+\frac{c}{4} \xi$. From this and (2.13) we see that $A R_{\xi}-R_{\xi} A=0$ on this subset. By Remark 2, it is contradictory. Hence, (5.8) is proved.

Using (5.3) and (5.8), we obtain

$$
A^{2} \xi=h A \xi+\frac{c}{4 \alpha^{2}}\left(h \alpha+\frac{c}{4}-3 \alpha^{2}\right) \xi,
$$

which enables us to obtain

$$
\alpha^{2}\left(\beta-h \alpha+\frac{3}{4} c\right)=\frac{c}{4}\left(h \alpha+\frac{c}{4}\right) .
$$

Further, from (5.5) we see that $\rho=h$ and hence

$$
\lambda=h-\alpha .
$$

Putting $X=\xi$ in (2.4) and using (5.5), we find

$$
S \xi=\left\{\frac{c}{2}(n-1)+h \alpha-\beta\right\} \xi .
$$

Differentiating this covariantly, we get

$$
\left(\nabla_{X} S\right) \xi+S \nabla_{X} \xi=X(h \alpha-\beta) \xi+\left\{\frac{c}{2}(n-1)+h \alpha-\beta\right\} \nabla_{X} \xi .
$$

If we replace $X$ by $\xi$ in this, and make use of (2.4) and $\nabla_{\xi} S=0$, then we have

$$
A^{2} U-h A U-\left(\beta-h \alpha+\frac{3}{4} c\right) U+\xi(h \alpha-\beta) \xi=0,
$$


which implies that

$$
\xi \beta=\xi(h \alpha) \text {. }
$$

Putting $X=U$ in (3.8) and using (3.3), (5.6) and (5.8), we have

$$
\alpha^{2} \nabla_{U} U=-\frac{c}{4} \mu(\xi \alpha) W+\alpha\left(\alpha \mu^{2}+\frac{c}{4} \lambda\right) U,
$$

which, together with (3.4) and (5.8) yields

$$
\alpha \nabla \alpha=\left(\alpha^{2}+\frac{3}{4} c\right) U+\frac{\xi \alpha}{\mu}\left(\alpha A W+\frac{c}{4} W\right) .
$$

Now, we are going to prove $\xi \alpha=0$ on $\Omega$. Since we have $\rho=h$, we can write (5.5) as $A^{2} \xi=h A \xi+(\beta-h \alpha) \xi$. Differentiating this covariantly and making use of the second equation of (2.1), we find

$$
\begin{aligned}
g\left(\left(\nabla_{X} A\right) A \xi, Y\right)+ & g\left(A\left(\nabla_{X} A\right) \xi, Y\right)+g\left(A^{2} \phi A X, Y\right)-h g(A \phi A X, Y) \\
= & (X h) g(A \xi, Y)+h g\left(\left(\nabla_{X} A\right) \xi, Y\right) \\
& +X(\beta-h \alpha) \eta(Y)+(\beta-h \alpha) g(\phi A X, Y)
\end{aligned}
$$

which together with $(2.3),(2.9)$ and (5.6) gives,

$$
\left(\nabla_{\xi} A\right) A \xi=h A U-\frac{c}{4} U+\frac{1}{2} \nabla \beta .
$$

If we replace $X$ by $\xi$ in (5.13) and use (2.9) and the last equation, then we get

$$
3 A^{2} U-2 h A U-\left(\beta-h \alpha+\frac{c}{4}\right) U=(\xi h) A \xi-A \nabla \alpha+h \nabla \alpha-\frac{1}{2} \nabla \beta,
$$

which, connected to (3.3) and (5.9) yields

$$
\frac{1}{2} \alpha^{2} \nabla \beta+\frac{c}{4}\left(2 \alpha^{2}+h \alpha+\frac{c}{2}\right) U=\alpha^{2}(\xi h) A \xi-\alpha^{2} A \nabla \alpha+h \alpha^{2} \nabla \alpha .
$$

Substituting (5.12) into this, we obtain

$$
\begin{aligned}
& \frac{1}{2} \alpha^{2} \nabla \beta+\left\{\frac{c}{4}\left(2 \alpha^{2}+h \alpha+\frac{c}{2}\right)-\left(h \alpha+\frac{c}{4}\right)\left(\alpha^{2}+\frac{3}{4} c\right)\right\} U \\
& =\alpha^{2}(\xi h) A \xi-\frac{\alpha}{\mu}(\xi \alpha)\left(\alpha A^{2} W+\frac{c}{4} A W\right)+\frac{h \alpha}{\mu}(\xi \alpha)\left(\alpha A W+\frac{c}{4} W\right) .
\end{aligned}
$$

Differentiating (5.9) and using itself, we find

$$
\alpha^{3}(Y \beta)-\alpha^{2}\left(\alpha^{2}+\frac{c}{4}\right) Y h=\left(h \alpha^{3}-\frac{c}{4} h \alpha-\frac{c^{2}}{8}\right) Y \alpha
$$


which implies that

$3 \alpha\{(X \alpha)(Y \beta)-(Y \alpha)(X \beta)\}-\left(3 \alpha^{2}+\frac{c}{4}\right)\{(X \alpha)(Y h)-(Y \alpha)(X h)\}=0$.

Combining (5.15) to this, we obtain $(X \alpha)(Y h)-(Y \alpha)(X h)=0$. This, together with (5.14) yields

$$
\xi \alpha\left\{\alpha^{2}(X h)-\left(h \alpha+\frac{c}{2}\right) X \alpha\right\}=0 .
$$

Thus, we have $\xi \alpha=0$. In fact, if not, then we have

$$
\alpha^{2}(X h)=\left(h \alpha+\frac{c}{2}\right) X \alpha
$$

on this subset. From this and (5.15) we see that $\alpha(X \beta)=\left(2 h \alpha+\frac{c}{2}\right) X \alpha$, which together with (5.8) gives $\alpha^{2}(U \beta)=\left(2 h \alpha+\frac{c}{2}\right)\left(\alpha^{2}+\frac{3}{4} c\right) \mu^{2}$. If we take the inner product $U$ to (5.14) and take account of the last equation, we obtain $2 \alpha^{2}+h \alpha+\frac{c}{2}=0$ on the subset, a contradiction by virtue of (5.4). Therefore, $\xi \alpha=0$ is established everywhere on $\Omega$.

From this fact, (5.4), (5.10) and (5.11) imply respectively to

$$
\xi h=0, \quad \xi \lambda=0, \quad \xi \beta=0 .
$$

Further, (5.12) becomes

$$
\alpha \nabla \alpha=\left(\alpha^{2}+\frac{3}{4} c\right) U
$$

Since we have $g\left(\nabla_{X} U, U\right)=\mu(X \mu)$, taking the inner product $U$ to (3.8) and making use of (3.3), (5.13) and the fact that $\xi \alpha=0$, we obtain $\alpha \nabla \mu=\mu \alpha U+\frac{c}{4} \phi A W$. This, together with (5.6) gives

$$
\frac{1}{2} \alpha \nabla \mu^{2}=\left(\alpha \mu^{2}+\frac{c}{4} \lambda\right) U
$$

Differentiating (5.18) covariantly and taking the symmetric part obtained, we find

$$
\frac{c}{4}\{(X \lambda) u(Y)-(Y \lambda) u(X)\}+\left(\alpha \mu^{2}+\frac{c}{4} \mu\right) d u(X, Y)=0,
$$

where we have used (5.17) and (5.18). If we replace $Y$ by $\xi$ to this, and make use of (5.16), we obtain

$$
\left(\alpha \mu^{2}+\frac{c}{4} \lambda\right) d u(X, \xi)=0 .
$$

Finally, we suppose that $\alpha \mu^{2}+\frac{c}{4} \lambda \neq 0$ on $\Omega$, and that we restrict the arguments on such a place. Then we have from the last equation

$$
g\left(\nabla_{\xi} U, X\right)+g\left(\nabla_{X} \xi, U\right)=0 .
$$


This, together with (2.9), (2.10), (5.6) and (5.10) imply that

$$
\phi(3 A U+\nabla \alpha)+\mu h W=0,
$$

which enables to obtain

$$
\nabla \alpha=h U-3 A U
$$

Thus, it follows that

$$
\alpha \nabla \alpha=\left(h \alpha+\frac{3}{4} c\right) U
$$

by virtue of (3.3). Comparing this with (5.17), we see that $h=\alpha$ and hence $\lambda=0$ because of (5.10). Therefore (5.9) is reduced to

$$
\alpha^{2} \mu^{2}+\frac{c}{2} \alpha^{2}=\left(\frac{c}{4}\right)^{2} .
$$

Further, (5.18) turns out to be $\nabla \mu=\mu U$.

Differentiating (5.19) along $\Omega$, and using (5.17) and the last equation, we obtain

$$
\left(2 \alpha^{2}+\frac{3}{4} c\right) \mu^{2}+\frac{c}{2}\left(\alpha^{2}+\frac{3}{4} c\right)=0,
$$

which, connected to (5.19) implies that $\alpha$ is constant. Hence, $\mu$ is constant, a contradiction because of the fact that $\nabla \mu=\mu U$. Consequently we have $\alpha \mu^{2}+\frac{c}{4} \lambda=0$ on $\Omega$, which together with (5.18) yield that $\nabla \mu=0$. Owing to Lemma $1, M$ is a Hopf hypersurface in $M_{n}(c)$. This completes the proof.

\section{References}

[1] J. Berndt, Real hypersurfaces with constant principal curvatures in complex hyperbolic spaces, J. Reine Angew. Math. 395 (1989), 132-141.

[2] J. Berndt and H. Tamaru, Cohomogeneity one actions on noncompact symmetric spaces of rank one, Trans. Amer. Math. Soc. 359 (2007), 3425-3438.

[3] T. E. Cecil and P. J. Ryan, Focal sets and real hypersurfaces in complex projective space, Trans. Amer. Math. Soc. 269 (1982), 481-499.

[4] J. T. Cho and U-H. Ki, Real hypersurfaces of a complex projective space in terms of Jacobi operators, Acta Math. Hungar. 80 (1998), 155-167.

[5] J. T. Cho and U-H. Ki, Real hypersurfaces in a complex space form with symmetric Jacobi Reeb flow, Canadian Math. Bull. 51 (2008), 359-371.

[6] U-H. Ki, H. Kurihara and R. Takagi, Jacobi operators along the structure flow on real hypersurfaces in a nonflat complex space form, to appear in Tsukuba J. Math.

[7] U-H. Ki and H. Liu, Some characterizations of real hypersurces of type (A) in a nonflat complex space form, Bull. Korean Math. Soc. 44 (2007), 152-172. 
[8] U-H. Ki, J. D. Pérez, F. G. Santos and Y. J. Suh, Real hypersurfaces in complex space forms with $\xi$-parallel Ricci tensor and structure Jacobi operator, J. Korean Math. Soc. 44 (2007), 307-326.

[9] U-H. Ki and Y. J. Suh, On real hypersurfaces of a complex space form, Math. J. Okayama Univ. 32 (1990), 207-221.

[10] N.-G. Kim, U-H. Ki and H. Kurihara, Characterizations of real hypersurfaces of type $A$ in complex space form used by the $\xi$-parallel structure Jacobi operator, Honam Math. J. 30 (2008), 535-550.

[11] S. Montiel and A. Romero, On some real hypersurfaces of a complex hyperbolic space, Geom Dedicata 20 (1986), 245-261.

[12] M. Okumura, On some real hypersurfaces of a complex projective space, Trans. Amer. Math. Soc. 212 (1975), 355-364.

[13] M. Ortega, J. D. Pérez and F. G. Santos, Non-existence of real hypersurfaces with parallel structure Jacobi operator in nonflat complex space forms, Rocky Mountain J. 36 (2006), 1603-1613.

[14] J. D. Pérez, F. G. Santos and Y. J. Suh, Real hypersurfaces in complex projective spaces whose structure Jacobi operator is D-parallel, Bull. Belg. Math. Soc. Simon Stevin 13 (2006), 459-469.

[15] R. Takagi, On homogeneous real hypersurfaces in a complex projective space, Osaka J. Math. 19 (1973), 495-506.

[16] R. Takagi, Real hypersurfaces in a complex projective space with constant principal curvatures I, II, J. Math. Soc. Japan 15 (1975), 43-53, 507-516.

Nam-Gil Kim

Department of Mathematics

Chosun University

Kwangju 501-759, Korea

E-mail : ngkim@chosun.ac.kr 